\title{
Traitement chirurgical de l'obésité - mise à jour de l'ordonnance sur les prestations de l'assurance des soins (OPAS)
}

\author{
A. Glättli*
}

* Président du Swiss Study Group
for Morbid Obesity

Correspondance:

Dr Andreas Glättli

Schänzlistrasse 33

CH-3013 Berne

E-mail: glaettli@bauchchirurgie.ch
L'annexe 1 de l'OPAS définit les conditions par lesquelles, en présence d'une obésité morbide, la prise en charge des interventions chirurgicales dont le but est la perte pondérale et l'amélioration des comorbidités associées à l'obésité constitue une prestation obligatoire pour les caissesmaladie. Le Swiss Study Group for Morbid Obesity (SMOB) se tient de puis sa fondation en 1996 à la disposition de l'Office fédéral des assurances sociales (OFAS) et actuellement de l'Office fédéral de la santé publique (OFSP). En tant que partenaire spécialisé, il joue un rôle de conseiller auprès de la Commission fédérale des prestations pour l'élaboration des recommandations concernant les prestations obligatoires dans le domaine du traitement chirurgical de l'obésité.

L'annexe 1 de l'OPAS concernant le traitement chirurgical de l'obésité, qui datait de 1983, a été mise à jour dans le cadre de cette collaboration, et sa nouvelle version est entrée en vigueur le $1^{\text {er }}$ janvier 2000. Elle était fondée essentiellement sur les conclusions de la «Conférence de consensus sur le traitement chirurgical de l'obésité en Suisse» [1], à laquelle le PD Dr F. Horber a très activement participé en tant que président du SMOB. Dans l'ensemble, cette nouvelle version de l'annexe 1 reprenait les critères reconnus sur le plan international pour le traitement chirurgical de l'obésité, à l'exception de l'indice de masse corporelle (Body Mass Index = BMI), dont la limite a été définie uniformément à $40 \mathrm{~kg} / \mathrm{m}^{2}$. Dans le reste du monde, la limite est aussi fixée à 40 , mais l'indication opératoire est également acceptée si le BMI est $\geq 35$ en présence de comorbidités sévères liées à l'obésité.

Pour des raisons restées inexpliquées jusqu'à présent, le mot «ininterrompu» a été introduit dans l'alinéa d) de l'annexe de l'OPAS (tab. 1). Ceci signifiait que la prise en charge pour les assurances-maladie n'était obligatoire que «pour autant qu'un traitement approprié ininterrompu de deux ans visant à la perte pondérale se soit révélé inefficace». De façon évidente pour le commun des mortels comme pour les spécialistes de l'obésité, un régime approprié ininterrompu de deux ans n'est pas réalisable. Cette modification de l'annexe aurait donc pu signifier que, pendant les deux années suivant son introduction, aucune intervention chirurgicale visant à la perte de poids n'aurait été possible. De fait, ce petit mot a eu pour conséquence le refus de la prise en charge d'une intervention médicalement indiquée chez plusieurs patients.

Ce changement a motivé plusieurs interventions. La Fondation Suisse de l'Obésité (FOSO), par la main de son président Monsieur von Grünigen, a protesté par écrit auprès de l'OFAS. Le SMOB aussi a exigé de l'OFSP que le mot «ininterrompu» soit supprimé de l'alinéa d). De plus, le SMOB a proposé des définitions permettant l'interprétation correcte des termes «approprié» et «inefficace». Le groupe d'entraide des patients bernois, par l'intermédiaire de Madame Emma Wüthrich, a lancé une collecte de signature pour soutenir cette demande. D'autres manifestations de soutien ont compris divers articles de presse et une émission sur une TV locale.

$\mathrm{Au}$ cours de nos interventions répétées, il s'est vite avéré que les intérêts du SMOB, qui représente les malades et les spécialistes du traitement de l'obésité, et ceux de la Commission fédérale des prestations, dont le souci prioritaire est le contrôle des coûts de la santé, n'étaient pas les mêmes. Des études toutes récentes ont pourtant confirmé que la chirurgie est bien supérieure aux thérapies conservatrices dans le traitement de l'obésité morbide [4]. Nous considérons donc comme un vrai succès le fait d'avoir obtenu une solution satisfaisante, et ceci avant tout pour le bien des malades.

Avec les différentes parties concernées, le SMOB exprime sa satisfaction de constater que, dans le dernière version de l'annexe 1 de l'OPAS, valable à partir du $1^{\text {er }}$ janvier 2005, le mot «ininterrompu» a été supprimé de l'alinéa d). Ceci contribuera à une diminution de la discrimination à laquelle les malades atteints d'obésité morbide sont soumis quotidiennement.

Le SMOB tient depuis le $1^{\text {er }}$ janvier 2001 un registre d'évaluation selon l'alinéa g) de l'annexe. Ce dernier collige toutes les opérations réalisées en Suisse dans le cadre du traitement chirurgical de l'obésité. Le SMOB analyse ces données et les transmet sous forme anonymisée 
Tableau 1

Conditions de l'annexe 1 de l'OPAS valables à partir du $1^{\text {er }}$ janvier 2005.

\begin{tabular}{|c|c|c|c|}
\hline Interventions & Prise en charge & Conditions & Valable dès \\
\hline \multirow{7}{*}{$\begin{array}{l}\text { Chirurgie de l'obésité (By-pass gastrique, } \\
\text { cerclage gastrique, gastroplastie verticale } \\
\text { calibrée) }\end{array}$} & \multirow[t]{7}{*}{ Oui } & a. Après en avoir référé au médecin conseil. & \multirow[t]{7}{*}{$1.1 .2000 / 1.1 .2004 / 1.1 .2005$} \\
\hline & & b. Le patient ne doit pas avoir plus de 60 ans. & \\
\hline & & c. Le patient doit avoir un BMI de plus de 40. & \\
\hline & & $\begin{array}{l}\text { d. Un traitement amaigrissant approprié de deux ans } \\
\text { est resté sans effets. }\end{array}$ & \\
\hline & & $\begin{array}{l}\text { e. Le malade doit en outre être porteur de l'une des } \\
\text { affections suivantes: Hypertension artérielle mesurée } \\
\text { avec une manchette large; diabète sucré; syndrome } \\
\text { des apnées du sommeil; dyslipidémie; affection } \\
\text { dégénérative invalidante de l'appareil locomoteur; } \\
\text { affection coronarienne; stérilité avec hyperandro- } \\
\text { génisme; syndrome des ovaires polykystiques chez } \\
\text { les femmes en âge de procréer. }\end{array}$ & \\
\hline & & $\begin{array}{l}\text { f. L'intervention doit être réalisée dans un centre } \\
\text { disposant d'une équipe multidisciplinaire expéri- } \\
\text { mentée dans le traitement de l'obésité (chirurgie, } \\
\text { psychothérapie, conseils nutritionnels, médecine } \\
\text { interne). }\end{array}$ & \\
\hline & & $\begin{array}{l}\text { g. Système d'évaluation uniforme fondé sur des } \\
\text { données quantitatives et une statistique des coûts. }\end{array}$ & \\
\hline
\end{tabular}

à l'OFSP. Depuis la création de ce registre, aucun centre n'est autorisé à pratiquer la chirurgie de l'obésité s'il ne participe pas à la tenue du registre par la transmission de ses données.

Afin d'éviter tout malentendu, le SMOB a fait des propositions concrètes pour l'interprétation de l'alinéa d):

Valent comme traitement adéquat:

1. conseils nutritionnels par les médecins (y compris les généralistes) ou un/une diététicien/ne diplomé(e) et/ou

2. traitement comportemental y compris la psychothérapie et/ou

3. régime hypocalorique et/ou

4. programme d'exercice physique et/ou

5. traitement médicamenteux;

6. un traitement approprié peut également être réalisé indépendamment de la couverture de l'assurance-maladie (p.ex. financé directement par le malade).

Traitement inefficace: un traitement amaigrissant approprié dont la durée totale (additive) est d'au moins deux ans est considéré comme inefficace si, 24 mois après son début, une perte pondérale minimale de $16 \%$ n'a pas été atteinte ou maintenue [5]. Ces deux années de traitement conservateurs peuvent se diviser en différents programmes appropriés répondant aux définitions ci-dessus. Pour être pris en compte, un segment thérapeutique doit durer au minimum un mois.

L'obésité, et avec elle les comorbidités qui lui sont associées, vont poser dans un futur proche un important problème économique et de politique de la santé publique en Suisse [6]. L'approche de cette problématique ne peut se faire que dans le cadre d'une vision interdisciplinaire. Le Swiss Study Group for Morbid Obesity continuera de se tenir à disposition des différents partenaires dans un esprit de dialogue constructif.

\section{Références}

1 Golay A, Burckhardt P, Bürgi U, de Marco D, Héraïef E, Horber F, Keller U, Laurent-Jaccard A, Noseda G, Schutz Y, Suter PM. Consensus sur le traitement de l'obésité en Suisse 1999. Schweiz Med Wochenschr 1999;129(Suppl 114):5S-36S.

2 NIH conference. Gastrointestinal surgery for severe obesity. Consensus Development Conference Panel. Ann Intern Med 1991;115(12):956-61.

3 Sauerland S, Angrisani L, Belachew M, Chevallier JM, Favretti F, Finer N, et al. Obesity surgery: evidencebased guidelines of the European Association for Endoscopic Surgery (EAES). Surg Endosc 2004; 19(2):200-21.

4 Sjostrom L, Lindroos AK, Peltonen M, Torgerson J, Bouchard C, Carlsson B, et al. Lifestyle, diabetes, and cardiovascular risk factors 10 years after bariatric surgery. N Engl J Med 2004;351:2683-93.

5 Sjostrom CD, Peltonen M, Wedel H, Sjostrom L. Differentiated long-term effects of intentional weight loss on diabetes and hypertension. Hypertension 2000;36(1):20-5.

6 sda. BAG-Studie: Übergewicht verursacht 2,7 Milliarden Franken Kosten. Bull Méd Suisses 2004; 85(40):2110. 